\title{
Are Shaded Fuelbreaks the Answer to Canada's Community Wildfire Protection Problem?
}

$\mathbf{O}_{\mathrm{d}}^{\mathrm{n}}$ n March 26, 2019, Bruce Blackwell delivered a presentation entitled "Consideration and Design of Landscape Level Fuelbreaks - A BC Example" as part of the CIF/IFC National Electronic Lecture Series (see below) that is deserving of comment.

First of all, as a matter of clarity, it is worth noting the distinction between the terms firebreaks and fuelbreaks, as they are often used interchangeably. A firebreak represents a barrier devoid of fuel (e.g., mineralized soil) designed to stop or halt a fire's spread. This is in contrast to a fuelbreak which is a strategically located wide strip where the vegetative fuels have been modified in order to reduce a fire's spread and intensity compared to the surrounding area so that wildfires can be controlled more safely and effectively. A shaded fuelbreak is built in forest areas where trees on the break are thinned and pruned to reduce the fire potential yet retain enough crown canopy to make a less favourable microclimate for surface fires. Surface fuels are commonly treated by some form of prescribed fire as part of their construction and of the maintenance program.

The concept of shaded fuelbreaks originated in the dry forest types of northern and central California back in the late 1950s. No research on shaded fuelbreaks has specifically been undertaken in Canada nor have any of the limited number of shaded fuelbreaks established to date been "tested" by wildfire in order to form an opinion of their relative effectiveness.

Blackwell acknowledged that shaded fuelbreaks were not a panacea for the wildland-urban interface fire problem and that they were incapable of stopping a wildfire burning under extreme fire weather conditions as a result of medium- and long-range spotting. However, given the expense and effort to construct them, expectations undoubtedly run high regardless of any cautionary statements that might be made otherwise. He also stated that

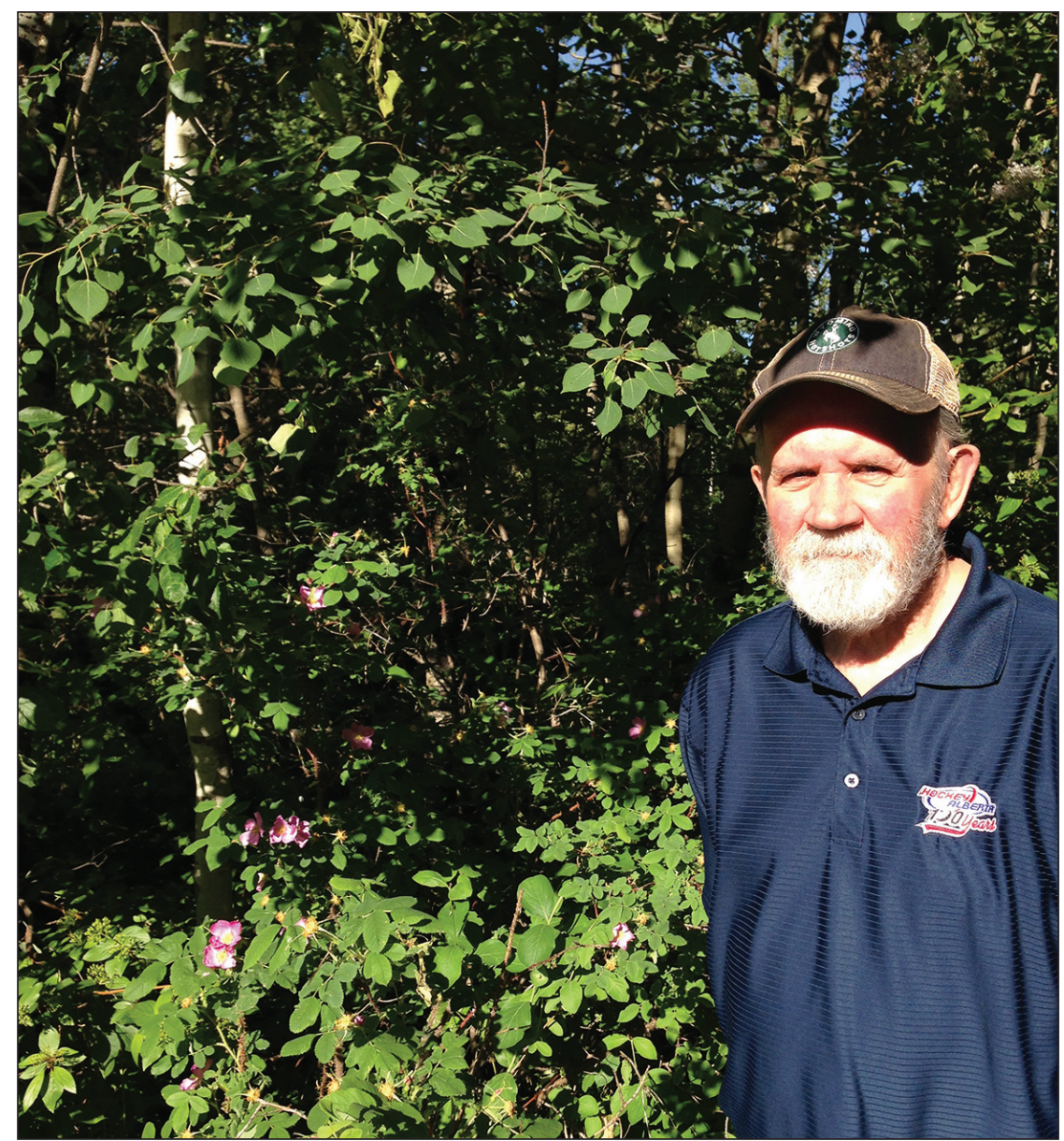

Martin E. Alexander, PhD, RPF

Proprietor, Wild Rose Fire Behaviour, Leduc County, AB/ Propriétaire, Wild Rose Fire Behaviour, Comté de Leduc, Alberta mea2@telus.net

while there was "theory" to support the application of shaded fuelbreaks (although he did not provide any specifics), research was still needed. His suggestion of a 50 tonne/ha residual surface fuel load seems alarming high. It is also unclear what his reference to a " $90^{\text {th }}$ percentile" state actually refers to.

Years ago, a good friend of mine, Rick Lanoville, remarked that "northern solutions are needed for northern problems". This remark has caused me to raise the question: Is the shaded fuel- break concept flawed in some way? Could it benefit from undertaking a detailed problem analysis involving both fire research and fire operations staff? There is much to consider before embarking on a wider expansion of shaded fuelbreaks to solve the community wildfire protection problem (e.g., effectiveness, cost-benefit analysis, firefighter safety concerns, suppression strategy and tactics, logistics, acceptable surface fuel loads, alternative types of fuelbreaks). 


\section{Les coupe-feux ombragés sont-ils vraiment la solution aux problèmes de protection contre les incendies de forêt des communautés canadiennes?}

e 26 mars 2019, Bruce Blackwell a - fait un exposé intitulé "Consideration and Design of Landscape Level Fuelbreaks - A BC Example» (Prise en compte et conception de coupe-feux au niveau du paysage - Un exemple en Colombie-Britannique) dans le cadre des conférences électroniques nationales de l'IFC/CIF (voir ci-dessous). Je crois qu'il mérite quelques commentaires.

Tout d'abord, par souci de clarté, il convient de distinguer les termes coupe-feu (firebreak) et coupe-feu sous ombragé (fuelbreak) ${ }^{1}$, car ils sont souvent utilisés comme synonymes. On appelle coupe-feu une barrière dépourvue de combustible (p. ex. : au sol minéral) conçue pour stopper ou arrêter la propagation d'un incendie. Il diffère du coupe-feu ombragé qui consiste en une large bande de terrain placée à un endroit stratégique dans laquelle les combustibles végétaux ont été modifiés afin de réduire la propagation et l'intensité d'un incendie par rapport à la zone limitrophe, de sorte que les incendies puissent être maîtrisés de façon plus

${ }^{1}$ NDLT : Il n'existe pas de mots pour décrire «fuelbreak» en français. Nous proposons celui-ci. sûre et avec plus d'efficacité. On aménage un coupe-feu ombragé en éclaircissant la bande boisée et en y élaguant les arbres pour réduire le risque d'incendie, tout en conservant suffisamment de couvert végétal pour créer un microclimat moins favorable aux feux de surface. En général on gère les combustibles de surface par une forme de brûlage dirigé lors de la création et de l'entretien des coupe-feu ombragés.

Le concept des coupe-feux ombragés est apparu dans les forêts sèches du nord et du centre de la Californie vers la fin des années 1950. On ne rapporte aucune étude spécifique sur les coupefeux ombragés au Canada et aucun des rares coupe-feux ombragés établis à ce jour n'a été « testé » lors d'un incendie de forêt pour qu'on puisse se faire une idée sur leur efficacité relative.

Sur la base d'observations à moyen et grande échelle, Blackwell a reconnu que les coupe-feux ombragés n'étaient pas une panacée pour le problème d'incendies survenant en milieu périurbain et qu'ils n'étaient pas en mesure d'arrêter un incendie de forêt dans des conditions météorologiques extrêmes. Cependant, compte tenu des dépenses et des efforts nécessaires pour les établir, les attentes sont inévitablement élevées en dépit des mises en garde à leur égard. Il a aussi déclaré que, même si l'utilisation des coupe-feux ombragés reposait sur des bases scientifiques (bien qu'il n'en ait fourni aucune preuve), il y avait toujours pour la recherche. Le seuil de 50 tonnes/ha de combustible qu'il a proposé nous semble étonnamment élevé. Il est également difficile de savoir ce que représente l'état de « $90^{\mathrm{e}}$ percentile » auquel il fait référence.

Il y a des années, mon bon ami Rick Lanoville m'a fait le commentaire suivant « trouvons des solutions nordiques aux problèmes du Nord ». Cette remarque m'a amené à me demander si le concept de coupe-feu ombragé n'était pas un peu faussé. Ne pourrait-on pas l'améliorer en faisant une étude détaillée de la problématique qui mettrait à contribution les chercheurs en incendies forestiers et les personnes qui combattent ces incendies? Il y a plusieurs enjeux à considérer avant d'entreprendre un déploiement à grande échelle des coupe-feux ombragés pour répondre au problème de protection contre les incendies forestiers dans les communautés (p. ex. : efficacité, analyse coûts-avantages, problèmes de sécurité des pompiers, stratégie et tactiques de suppression, logistique, seuils acceptables de combustible de surface, autres types de coupe-feux). 


\section{Upcoming e-lectures/ Toutes les conférences électroniques à venir}

11 upcoming e-lectures are listed on the e-lecture page http://www.cififc.org/e-lectures/: as well as on the events calendar http://www.cif-ifc.org/ upcoming-events/.

Starting May $1^{\text {st }}$, the organizers are taking a spring/summer break to plan sessions/series for this fall. You can go on the calendar to view all the previous sessions this past year. These include series in collaboration with the Canadian Forest Service, Ducks Unlimited Canada and Healthy Forest Partnership. They are open access and the recordings can be viewed by non-members: http://www.cif-ifc.org/opensource-electures/.

The CIF/IFC has recently transitioned to an online-based registration system and participants (both-members and non-members) can now register by following the instructions below. The new system provides participants with calendar invitations and email reminders.

Toutes les conférences électroniques à venir sont répertoriées sur la page de conférences électroniques http://www. cif-ifc.org/e-lectures/: ainsi que sur le calendrier des événements http://www. cif-ifc.org/upcoming-events/.

À compter du $1^{\text {er }}$ mai, les organisateurs feront relâche pour le printemps et l'été afin de planifier les ateliers et les séries de cet automne. Vous pouvez consulter le calendrier pour voir tous les ateliers de l'année écoulée. Vous y trouverez les séries présentées en colla- boration avec le Service canadien des forêts, Canards Illimités Canada et Partenariat pour une forêt en santé. Ils sont disponibles sans frais et les enregistrements peuvent être visionnés par des non-membres: http://www.cififc.org/open-source-electures/

L'IFC/CIF a récemment adopté un système d'adhésion en ligne et les participants (membres et non-membres) peuvent désormais s'inscrire en suivant les instructions ci-dessous. Le nouveau système offre aux participants des annonces sur les événements à venir et des rappels par courriel.
To register for upcoming session(s):

- Go to the E-Lecture Catalogue

- Upcoming E-Lectures will be listed under "Upcoming Events"

- To register for a specific session(s), click the "Register" button below the session overview. For more information on a specific session(s), click the "Event Details" button.

- After clicking the "Register" button, please enter your name and preferred email address.

- Once registered, an email will be sent out to you providing the participation instructions.

\section{Pour vous inscrire aux prochains ateliers}

- Aller sur: Go to the E-Lecture Catalogue

- Les conférences électroniques à venir apparaîtront sous la rubrique « Upcoming Events »

- Pour vous inscrire à un ou des atelier(s), cliquer sur le bouton « Register » situé sous l'aperçu de l'atelier « session overview ». Pour plus de renseignements sur un ou des atelier(s) en particulier, cliquer sur le bouton « Event Details».

- Après avoir cliqué sur le bouton « Register », veuillez inscrire votre nom et l'adresse de courriel de votre choix.

- Une fois que vous serez inscrit, vous recevrez un courriel donnant les directives pour participer. 